\title{
Survey of Adult Influenza Vaccination Practices and Perspectives Among US Primary Care Providers (2016-2017 Influenza Season)
}

\author{
Jessica R. Cataldi, MD, MSCS ${ }^{1,2}$, Sean T. O'Leary, MD, MPH',2, Megan C. Lindley, MPH', \\ Laura P. Hurley, MD, MPH ${ }^{1,4}$, Mandy A. Allison, MD, MPH', , \\ Michaela Brtnikova, PhD, MPH ${ }^{1,2}$, Brenda L. Beaty, MSPH' , Lori A. Crane, PhD, MPH ${ }^{7.5}$, \\ and Allison Kempe, $\mathrm{MD}, \mathrm{MPH}^{1,2}$
}

\begin{abstract}
'Adult and Child Consortium for Health Outcomes Research and Delivery Science, University of Colorado Anschutz Medical Campus and Children's Hospital Colorado, Aurora, CO, USA; ${ }^{2}$ Department of Pediatrics, University of Colorado Anschutz Medical Campus, Aurora, CO, USA; ${ }^{3}$ National Center for Immunization and Respiratory Diseases, Centers for Disease Control and Prevention, Atlanta, GA, USA; ${ }^{4}$ Division of General Internal Medicine, Denver Health, Denver, CO, USA; ${ }^{5}$ Department of Community and Behavioral Health, Colorado School of Public Health, University of Colorado Anschutz Medical Campus, Aurora, CO, USA.
\end{abstract}

BACKGROUND: Seasonal influenza vaccination is recommended for all adults; however, little is known about how primary care physicians can communicate effectively with patients about influenza vaccination.

OBJECTIVE: To assess among general internal medicine (GIM) and family physicians (FP) regarding adult influenza vaccination: (1) recommendation and administration practices, (2) barriers to discussing and perceived reasons for patient refusal, and (3) factors associated with physician self-efficacy in convincing patients to be vaccinated. DESIGN: Email and mail survey conducted in FebruaryMarch 2017

PARTICIPANTS: Nationally representative sample of GIM and FP

MAIN MEASURES: Factor analysis was used to group similar items for multivariable analysis of barriers and strategies associated with high physician self-efficacy about convincing patients to be vaccinated (defined as disagreeing that they could do nothing to change resistant patients' minds). KEY RESULTS: Response rate was 67\% (620/930). Ninetyeight percent always/almost always recommended influenza vaccine to adults $\geq 65$ years, $90 \%$ for adults $50-64$ years, and $75 \%$ for adults $19-49$ years. Standing orders (76\%) and electronic alerts (64\%) were the most commonly used practice-based immunization strategies. Frequently reported barriers to discussing vaccination were other health issues taking precedence (41\%), time (29\%), and feeling they were unlikely to change patients' minds (24\%). Fifty-eight percent of physicians reported high self-efficacy about convincing patients to be vaccinated; these providers reported fewer patient belief barriers contributing to vaccine refusal $(\mathrm{RR}=0.93$ per item; 95\% CI (0.89-0.98); Cronbach's $\alpha=$ $0.70)$, were more likely to report using both fact- (1.08/item; (1.03-1.14); 0.66) and personal experience-based (1.07/

\begin{tabular}{l}
\hline \hline Electronic supplementary material The online version of this article \\
(https://doi.org/10.1007/s11606-019-05164-7) contains supplementary \\
material, which is available to authorized users.
\end{tabular}

Received January 31, 2019

Revised April 30, 2019

Accepted June 12, 2019

Published online July 19, 2019 item; (1.003-1.15); 0.65) communication strategies, and were more likely to work in practices using patient reminders for influenza vaccine (1.32; (1.16-1.50)).

CONCLUSIONS: Physicians identified barriers to successfully communicating about adult influenza vaccination but few effective strategies to counter them. Interventions to promote self-efficacy in communication and under-utilized practice-based immunization strategies are needed.

KEY WORDS: influenza; vaccination; physician communication; immunization delivery.

J Gen Intern Med 34(10):2167-75

DOI: $10.1007 / \mathrm{s} 11606-019-05164-7$

(C) Society of General Internal Medicine 2019

\section{BACKGROUND}

Influenza contributes to approximately 40,000 deaths annually in the USA. ${ }^{1}$ During the 2017-2018 influenza season, the cumulative hospitalization rate for illness associated with laboratoryconfirmed influenza was 106.6 per 100,000 for all age groups and was 460.9 per 100,000 among adults aged $\geq 65$ years. $^{2}$ The Advisory Committee on Immunization Practices (ACIP) recommends routine annual influenza vaccination for all people over 6 months of age who do not have contraindications ${ }^{3}$, yet influenza vaccination coverage remains well below Healthy People 2020 goals of $70 \%{ }^{4}$, with an estimated $37 \%$ of adults receiving the vaccine during the 2017-2018 influenza season. ${ }^{5}$

Patient barriers to adult influenza vaccination include lack of knowledge of vaccine recommendations, vaccine safety concerns, and negative attitudes toward influenza vaccination..$^{6-9}$ The Centers for Disease Control and Prevention (CDC) and the National Vaccine Advisory Committee recommend use of evidence-based strategies for adult immunization delivery including reminder/recall and immunization information system (IIS). ${ }^{10-13}$ The recently updated Standards of Adult Immunization Practice includes a statement that providers should 
communicate a strong recommendation for needed vaccines, yet there are not specific evidence-based communication recommendations for adult immunizations. ${ }^{13,}{ }^{14}$ Physician attitudes about influenza vaccination have been described; however, studies of how physicians communicate with patients about influenza vaccination and whether this communication is effective are lacking.

The objectives of this study were to examine the following among family physicians (FP) and general internal medicine (GIM) physicians: (1) practices regarding recommending and administering adult influenza vaccination, (2) perceived barriers to discussing and reasons for patient refusal of adult influenza vaccination, (3) provider communication strategies related to influenza vaccination, and (4) factors associated with physician self-efficacy in convincing patients to receive influenza vaccine.

\section{METHODS}

\section{Study Setting}

We administered an Internet and mail survey in February-March 2017 to a national network of physicians who spent $\geq 50 \%$ of their time practicing primary care. The Colorado Multiple Institutional Review Board reviewed and approved this study as an exempt research not requiring written informed consent.

\section{Study Population}

This survey was conducted as part of the Vaccine Policy Collaborative Initiative, a collaboration with the CDC to execute surveys to assess physician attitudes about vaccine issues. We recruited GIM and FP providers from the American College of Physicians (ACP) and the American Academy of Family Physicians (AAFP) to develop a network of primary care physicians. We performed quota sampling ${ }^{15}$ to ensure this network of physicians was similar to ACP and AAFP memberships for the characteristics of urban versus rural location, region, and practice setting. We have previously demonstrated that responses from network physicians were similar to those of physicians sampled randomly from American Medical Association physician databases with regard to demographics, practice characteristics, and attitudes about vaccination. ${ }^{15}$

\section{Survey Design}

We developed the survey in collaboration with CDC and with input from an expert in survey methodology (co-author LC). Survey items included new and previously developed questions incorporating knowledge from existing publications on influenza vaccination practices (survey available as Online Appendix). A national advisory panel of physicians pretested the survey. We used 4-point scales to assess the following: how often a physician recommended the influenza vaccine and how often patients agreed to receive the vaccine when recommended ("Rarely/Never" to "Almost always/always"); barriers to discussing influenza vaccine ("Major barrier" to "Not at all a barrier"); how much various factors contribute to influenza vaccine refusal ("Not at all" to "A lot"); whether respondents thought they might change a patient's mind about vaccination ("Strongly agree" to "Strongly disagree"); how often physicians use different communication strategies ("Never" to "Often or always"); and physician perception of each strategy's effectiveness ("Not at all effective" to "Very effective"). Finally, we asked physicians whether their practice uses specific strategies to facilitate influenza vaccine delivery (i.e., vaccination alerts/reminders, standing orders).

\section{Survey Administration}

The survey was sent over the Internet (Verint, Melville, NY, http://www.verint.com) or by mail, depending on physician preference. Following methods used in prior surveys ${ }^{16}$, we sent the Internet group an initial email message with up to eight email reminders, and we sent the mail group an initial mailing and up to two additional reminders. Non-respondents from the Internet group were sent up to two mail surveys in case of problems with email correspondence. We patterned the mail protocol on Dillman's Tailored Design Method. ${ }^{17}$

\section{Statistical Analysis}

We pooled Internet and mail survey responses for analyses because studies have found that physician attitudes are similar when obtained by either method. ${ }^{17-19}$ Characteristics of nonrespondents were obtained from the recruitment survey for the sentinel physician networks. We compared respondents and non-respondents and GIM and FP responses using $t$ tests, Wilcoxon tests, and chi-squared tests, as appropriate. Because results were generally not statistically different, combined results for GIM and FP are presented with exceptions noted.

We conducted a multivariable analysis with the dependent variable of physician self-efficacy about convincing patients to receive influenza vaccine by comparing responses to the statement, "When an adult patient refuses an influenza vaccine, there is not much I can do to change their mind." Those who somewhat/strongly agreed were labeled as having low selfefficacy while those who somewhat/strongly disagreed were labeled as having high self-efficacy. In the context of health behavior theories, self-efficacy is described as a person's selfperceived confidence and competence in enacting a behavior and is associated with engaging in that behavior. ${ }^{20,21}$ Focusing on the behavior of successful physician communication about influenza vaccination, we sought to identify factors associated with high self-efficacy for this behavior. Independent variables assessed for association with physician self-efficacy included physician specialty, practice location, provider communication strategies and practice-level immunization delivery strategies, perceived provider barriers to communication, and reasons for patient refusal of influenza vaccine. Bivariate analysis was conducted first for each independent variable and a cutoff of $p<0.25$ was used for inclusion in the multivariable model. When two survey items evaluated very similar content and were highly correlated, one representative item was chosen for inclusion in the model. For perceived barriers to communication, survey items that directly 
reflected a respondent's concern about his/her own communication abilities were deemed circular in nature and excluded from multivariable analysis.

Prior to building the multivariable model, factor analysis was performed to group similar items in the categories of perceived reasons for patient refusal and provider communication strategies, because each of these categories had $\geq 5$ items significantly associated with the outcome in bivariate analysis. Factor analysis resulted in one scale for reasons for patient refusal including four items reflecting patient suspicions or misinformation about influenza vaccine (Cronbach's alpha $=0.70$ ) and two scales for provider communication strategies with one scale of five items reflecting fact-based strategies (Cronbach's alpha $=0.66$ ) and a second scale of three items reflecting personal experience-based strategies (Cronbach's alpha $=0.65$ ). Scales were constructed for each factor by counting the number of items in each factor to which the respondent answered "Sometimes" or "Often or Always" for communication strategies or "Some" or "A lot" for reasons for patient refusal. Because the outcome of high self-efficacy in convincing patients to be vaccinated was common, we used a Poisson regression with robust error variance (SAS PROC GENMOD) to estimate risk ratios instead of odds ratios. We used a backward elimination procedure in which the least significant predictor in the model was eliminated sequentially. At each step, estimates were checked to ensure other variables were not affected by dropping the least significant variable. This resulted in retention of only those factors that were significant at $p<0.05$ in the final model. We left physician specialty in the model even though it was non-significant. Analyses were performed using SAS, version 9.4 (SAS Institute, Cary, NC).

\section{RESULTS}

\section{Survey Response and Respondent Characteristics}

The response rate was $67 \%$ overall (620/930), 61\% among GIM (283/466), and 73\% among FP (337/464). Compared with nonrespondents, respondents were more likely to be from the West and less likely to be from the South (Table 1). Providers of female

Table 1 Comparison of Respondents and Non-respondents and Additional Characteristics of Family Physician (FP) and General Internal Medicine (GIM) Physician Respondents

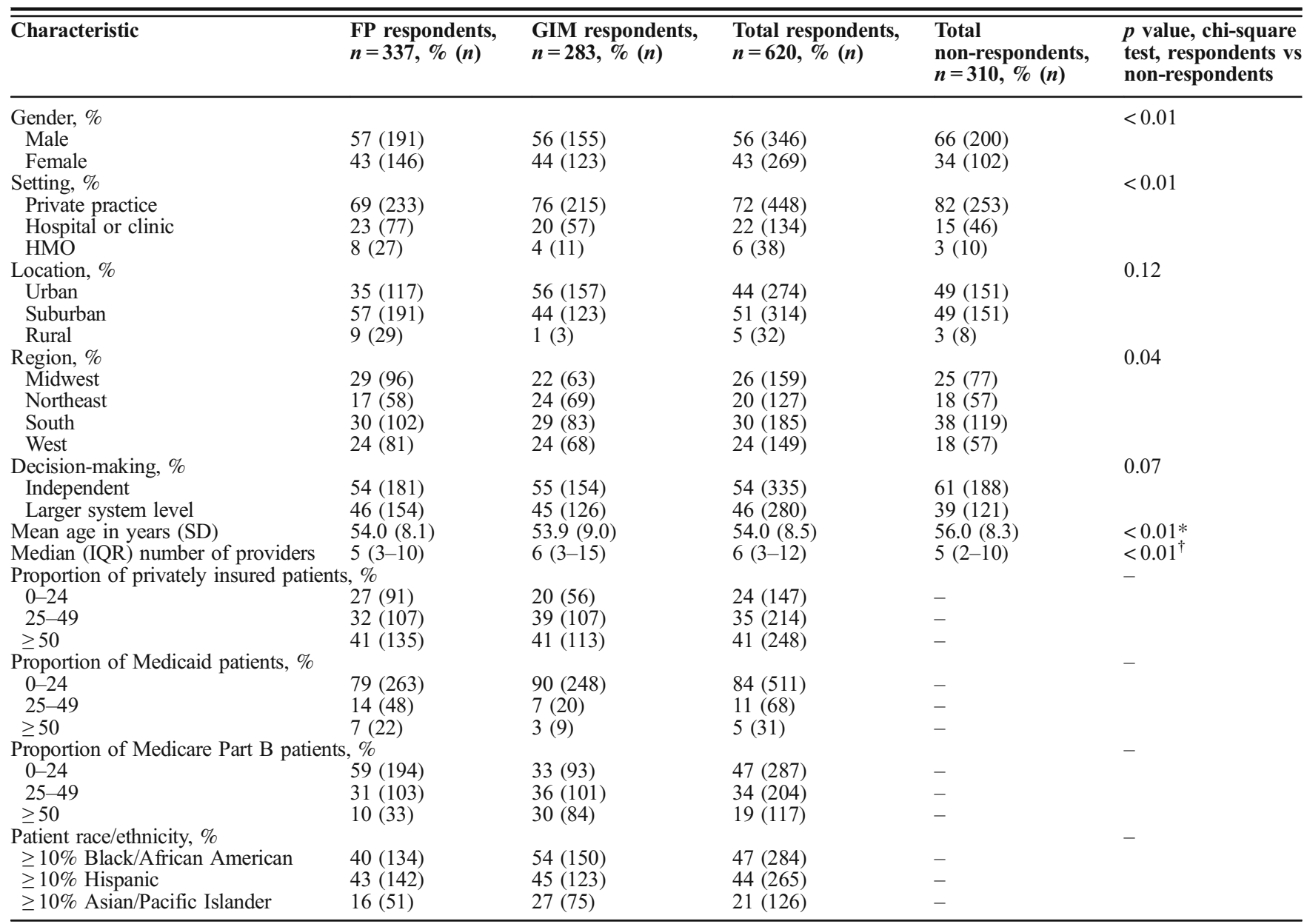


Table 2 Use of Practice-Level Strategies for Influenza Vaccination Delivery

\begin{tabular}{ll}
\hline \hline Practice-level strategy & $\begin{array}{l}\text { Used in practice, } N= \\
\mathbf{6 0 3} \%(\boldsymbol{n})\end{array}$ \\
\hline $\begin{array}{l}\text { Standing orders for influenza vaccination, } \\
\text { meaning a nurse or medical assistant can } \\
\text { administer influenza vaccine without talking }\end{array}$ & $76(459)^{*}$ \\
$\begin{array}{l}\text { with a provider first } \\
\text { A written or electronic alert that an adult } \\
\text { patient has not received influenza vaccine }\end{array}$ & $64(384)$ \\
$\begin{array}{l}\text { A state or regional IIS is used to record } \\
\text { influenza vaccination(s) an adult patient } \\
\text { receives in your practice }\end{array}$ & $41(248)^{*}$ \\
$\begin{array}{l}\text { Written, telephone, or email vaccination } \\
\text { reminders are sent to all adult patients in the } \\
\text { practice who are due for influenza vaccine }\end{array}$ & $35(212)^{\dagger}$ \\
A state or regional IIS is used to assess an \\
adult patient's influenza vaccination status \\
$\begin{array}{l}\text { Written, telephone, or email vaccination } \\
\text { reminders are sent to sub-groups of high-risk } \\
\text { adult patients in the practice who are due for } \\
\text { influenza vaccine }\end{array}$ \\
$\begin{array}{l}\text { Physicians are provided a list showing them } \\
\text { when all of their adult patients are due or } \\
\text { overdue for influenza vaccination } \\
\begin{array}{l}\text { Physicians are provided a list showing them } \\
\text { when sub-groups of high-risk adult patients in } \\
\text { the practice are due for influenza vaccination }\end{array}\end{array}$ \\
\hline
\end{tabular}

*Proportion of respondents using this strategy differed significantly between FP and GIM with more FP respondents using the strategy

${ }^{\dagger}$ Proportion of respondents using this strategy differed significantly between FP and GIM with more GIM respondents using the strategy gender, younger age, practicing in a hospital or clinic, and working in a practice with more providers were also more likely to respond to the survey.

\section{Physician Practices in Recommending and Administering Adult Influenza Vaccine}

Ninety-eight percent of respondents always or almost always recommended influenza vaccine to adults $\geq 65$ years, while $90 \%$ always/almost always recommended to adults 50-64 years old and $75 \%$ to adults $19-49$ years old. Sixty-six percent of physicians reported that adults $\geq 65$ years always/almost always agreed to get the influenza vaccine when they recommended it, compared with $29 \%$ for $50-64$-year-olds and $10 \%$ for 19-49-year-olds.

The most common practice-level strategies for adult influenza vaccination were use of standing orders and electronic alerts (Table 2). Less common strategies were providing physicians a list of patients needing influenza vaccine and assessing vaccination with an IIS. FP were more likely than GIM to use an IIS to assess influenza vaccination status (38\% vs $25 \%$; $p<0.001)$, to use an IIS to record influenza vaccination status (47\% vs $35 \% ; p=0.003)$, and to use standing orders for influenza vaccination $(80 \%$ vs $72 \% ; p=0.03)$. GIM physicians were more likely than FP to use written,

\section{$\square$ Major or moderate barrier $\quad \square$ Minor barrier $\quad \square$ Not at all a barrier}

Other health issues talking precedence over discussion of influenza vaccine risks and benefits*

The amount of time it takes

Feeling that I am unlikely to change patients' minds about their decision whether to get influenza vaccine

Adult patients will not understand information about risks and benefits*

Not knowing how to communicate with a patient who is resistant to getting influenza vaccine**

Not knowing enough about existing evidence regarding influenza vaccine safety

Not feeling well prepared to address unanticipated questions that patients raise about influenza vaccine

Not being knowledgeable enough about the severity of influenza**

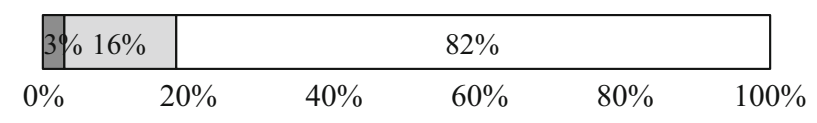

Fig. 1 Perceived Barriers to Discussing Influenza Vaccination with Patients. *FP more likely to report these items as major/moderate barriers $(p<0.05)$. ${ }^{+}$GIM more likely to report these items as major/moderate barriers $(p<0.05)$. 
telephone, or email reminders sent to adult patients who are due for influenza vaccination ( $40 \%$ vs $31 \% ; p=0.01)$.

\section{Perceived Barriers to Adult Influenza Vaccination}

The most frequently reported barriers to routinely discussing risks and benefits of influenza vaccine were other health issues taking precedence over vaccine discussion ( $41 \%$ reported as a major/moderate barrier), the amount of time required (29\%), and physicians' feeling they are unlikely to change patients' minds (23\%) (Fig. 1). The factors that physicians most frequently reported as contributing to influenza vaccine refusal among adult patients were belief that influenza vaccine will "make them sick" (57\% reported this contributes "a lot"), belief that the influenza vaccine causes "the flu" (38\%), and belief that they are unlikely to get influenza (33\%) (Fig. 2).

\section{Strategies for Addressing Refusal of Influenza Vaccine}

The strategies that physicians report using most with patients who initially refuse influenza vaccine include a statement that they (the physician) have received their influenza vaccine (63\% often or always use this strategy), discussion of influenza morbidity and mortality (56\%), discussion of the importance of being vaccinated in order to protect others who are close to the patient $(55 \%)$, a statement that it is safer to vaccinate than not to vaccinate (54\%), discussion of the effectiveness of influenza vaccine in preventing disease (54\%), and discussion of their own experience with influenza vaccine safety (53\%) (Table 3).

Few physicians reported any of these strategies as being "very effective" at convincing patients to receive influenza vaccine; however, many strategies were thought to be "somewhat effective" (Table 3). A discussion of the importance of

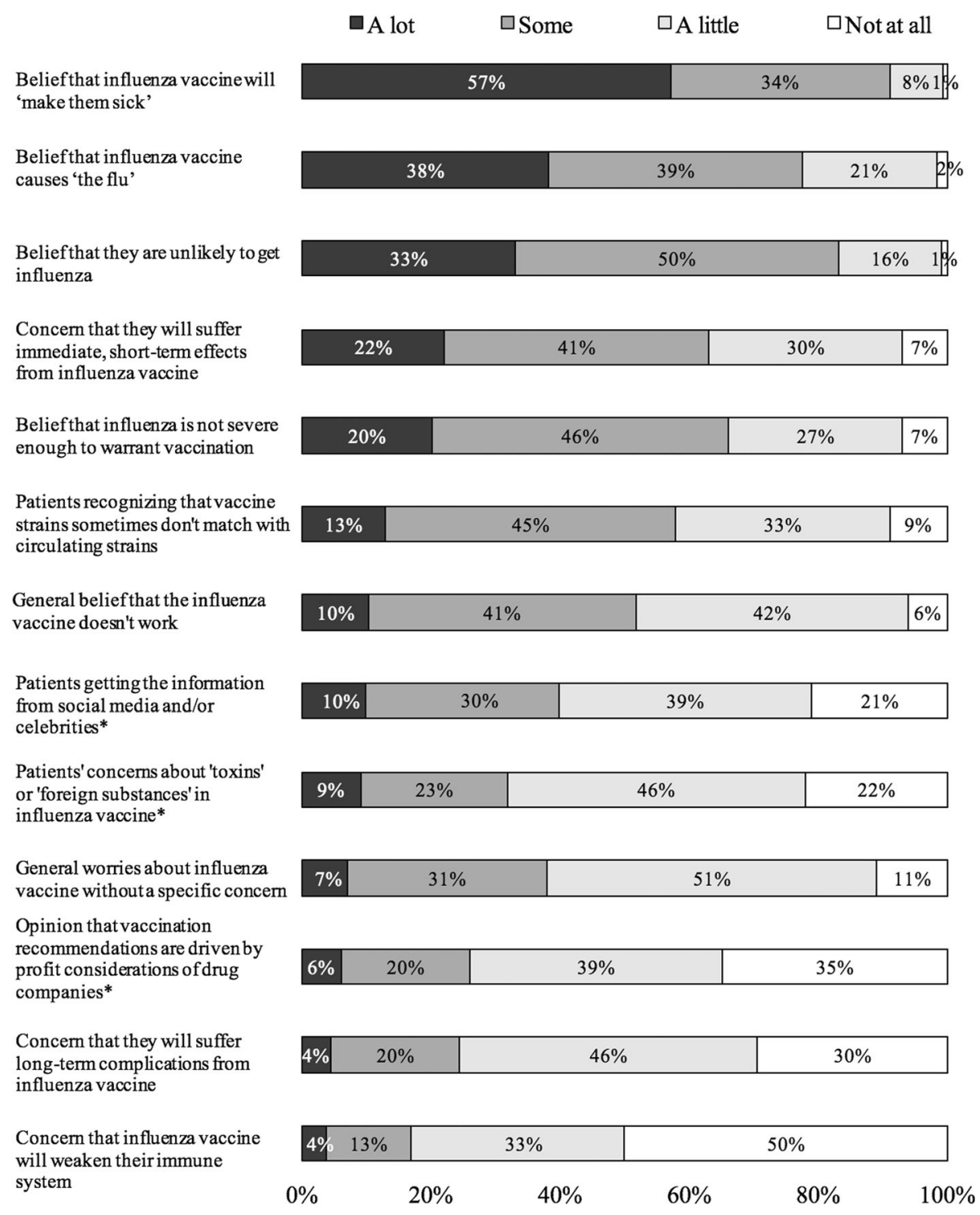

Fig. 2 Perceived Barriers Contributing to Influenza Vaccine Refusal Among Adult Patients. *FP more likely to report these items as contributing a lot or some to influenza vaccine refusal $(p<0.05)$. 
Table 3 Strategies Used and Perceived Effectiveness of Strategies for Communication about Influenza Vaccination

\begin{tabular}{|c|c|c|c|c|c|c|}
\hline \multirow[t]{2}{*}{ How often do you: } & \multicolumn{3}{|c|}{ Frequency used, $n=604, \%(n)$} & \multicolumn{3}{|c|}{ Perceived effectiveness, $n=594, \%(n)$} \\
\hline & $\begin{array}{l}\text { Often/ } \\
\text { always }\end{array}$ & Sometimes & $\begin{array}{l}\text { Never/ } \\
\text { rarely }\end{array}$ & $\begin{array}{l}\text { Very } \\
\text { effective }\end{array}$ & $\begin{array}{l}\text { Somewhat } \\
\text { effective }\end{array}$ & $\begin{array}{l}\text { Not very/not } \\
\text { at all effective }\end{array}$ \\
\hline $\begin{array}{l}\text { Use a statement from you that you received your influenza } \\
\text { vaccine }\end{array}$ & $63(377)$ & $25(147)$ & $13(76)$ & $13(78)$ & $54(316)$ & $33(194)$ \\
\hline $\begin{array}{l}\text { Use discussion of morbidity and mortality associated with } \\
\text { influenza }\end{array}$ & $56(338)$ & $38(228)$ & $6(36)$ & $8(48)$ & $70(415)$ & $22(131)$ \\
\hline $\begin{array}{l}\text { Use discussion of the importance of being vaccinated in } \\
\text { order to protect others who are close to the patient and at } \\
\text { high risk of influenza disease, such as a grandparent or a } \\
\text { grandchild }\end{array}$ & $55(333)$ & $38(231)$ & $6(38)$ & $24(144)$ & $59(351)$ & $17(98)$ \\
\hline $\begin{array}{l}\text { Use discussion of effectiveness of influenza vaccine in } \\
\text { preventing disease }\end{array}$ & $54(321)$ & $41(243)$ & $6(36)$ & $8(47)$ & $63(371)$ & $29(172)$ \\
\hline $\begin{array}{l}\text { Use a statement from you that you think it is safer to } \\
\text { vaccinate than not to vaccinate }\end{array}$ & $54(321)$ & $31(183)$ & $16(95)$ & $7(44)$ & $57(336)$ & $35(208)$ \\
\hline $\begin{array}{l}\text { Use discussion of your own experience with influenza } \\
\text { vaccine safety in your practice }\end{array}$ & $53(319)$ & $34(205)$ & $13(79)$ & $14(83)$ & $60(352)$ & $26(151)$ \\
\hline $\begin{array}{l}\text { Use discussion of your personal experiences with } \\
\text { influenza in your patients }\end{array}$ & $34(202)$ & $42(256)$ & $24(145)$ & $11(64)$ & $54(312)$ & $35(203)$ \\
\hline $\begin{array}{l}\text { Use discussion of the importance of high vaccination rates } \\
\text { in order to decrease influenza in the community (herd } \\
\text { immunity) }\end{array}$ & $28(170)$ & $31(187)$ & $41(244)$ & $5(27)$ & $32(188)$ & $63(372)$ \\
\hline $\begin{array}{l}\text { Use specific data from studies about the likelihood of side- } \\
\text { effects and adverse events associated with influenza } \\
\text { vaccination }\end{array}$ & $13(79)$ & $34(206)$ & 53 (319) & $3(15)$ & 39 (223) & $58(331)$ \\
\hline Refer patients to pro-vaccine websites & $2(11)$ & $9(56)$ & $89(533)$ & $1(5)$ & $15(85)$ & $84(468)$ \\
\hline
\end{tabular}

being vaccinated in order to protect others who are close to the patient and at high risk of influenza disease was perceived as the most effective strategy.

\section{Characteristics Associated with Provider Self- Efficacy for Successful Communication About Adult Influenza Vaccine}

Fifty-eight percent of respondents somewhat (42\%) or strongly $(16 \%)$ disagreed with the statement "When an adult patient refuses an influenza vaccine, there is not much I can do to change their mind." These respondents were classified as having high self-efficacy in convincing patients to receive influenza vaccination (Table 4). There were no differences in physician specialty or provider or practice demographic characteristics between physicians with high or low self-efficacy.

Several differences between providers with high and low self-efficacy remained after adjustment using multivariable analysis. Compared with providers with low self-efficacy, those with high self-efficacy were more likely to view adult patients not understanding information about risks and benefits as a minor barrier or not a barrier at all $(\mathrm{RR}=1.42 ; 95 \% \mathrm{CI}$ (1.06-1.89)). Providers with high self-efficacy reported fewer perceived patient barriers related to suspicions or misinformation about influenza vaccine as contributing "Some" or "A lot" to vaccine refusal ( $R R=0.93$ per item; $(0.89-0.98)$ ). Providers with high self-efficacy were also more likely to report using both fact-based ( $R R=1.08$ per item; $(1.03-1.14))$ and personal experience-based (1.07 per item; (1.003-1.15)) strategies "Sometimes" or "Often or Always." Physicians with high selfefficacy were more likely to work in practices where written, telephone, or email reminders were sent to all adult patients who were due for influenza vaccine $(R R=1.32 ;(1.16-1.50))$.

\section{CONCLUSIONS}

Our data show that physician recommendation for and patient acceptance of adult influenza vaccination differ by patient age group. Most respondents report use of some practice-level strategies for influenza vaccine delivery, but few use an IIS. Physicians endorsed a variety of barriers to discussing influenza vaccine and factors contributing to patient refusal of the vaccine. Most physicians were classified as having highefficacy based on their perception that they could change a patient's mind in cases of vaccine refusal. They reported using many different communication strategies to discuss influenza vaccine, yet they found few methods to be very effective. Providers with high self-efficacy differed from low selfefficacy providers in perceived barriers to influenza vaccination and in the communication and practice-level immunization strategies they use.

Primary care physicians use a variety of communication strategies with adult patients about influenza vaccine; however, they find few messages effective. A strategy that physicians perceived as most effective was discussing vaccination as a way to protect people close to the patient. This strategy adapts the language of herd immunity to a more personal message about protecting family members or close contacts from influenza. Respondents to our survey perceived the discussion of vaccination protecting a patient's family to be effective but perceived discussion of herd immunity in the community as less effective. In a survey of university students, more than $70 \%$ of unvaccinated respondents agreed that receiving information about protecting vulnerable individuals would make them more likely to receive the influenza vaccine, and a recent survey in Minnesota showed that educating respondents who were not previously knowledgeable about herd immunity 
Table 4 Factors Associated with Physician Self-efficacy in Convincing Patients to Receive Influenza Vaccination $(N=585)$

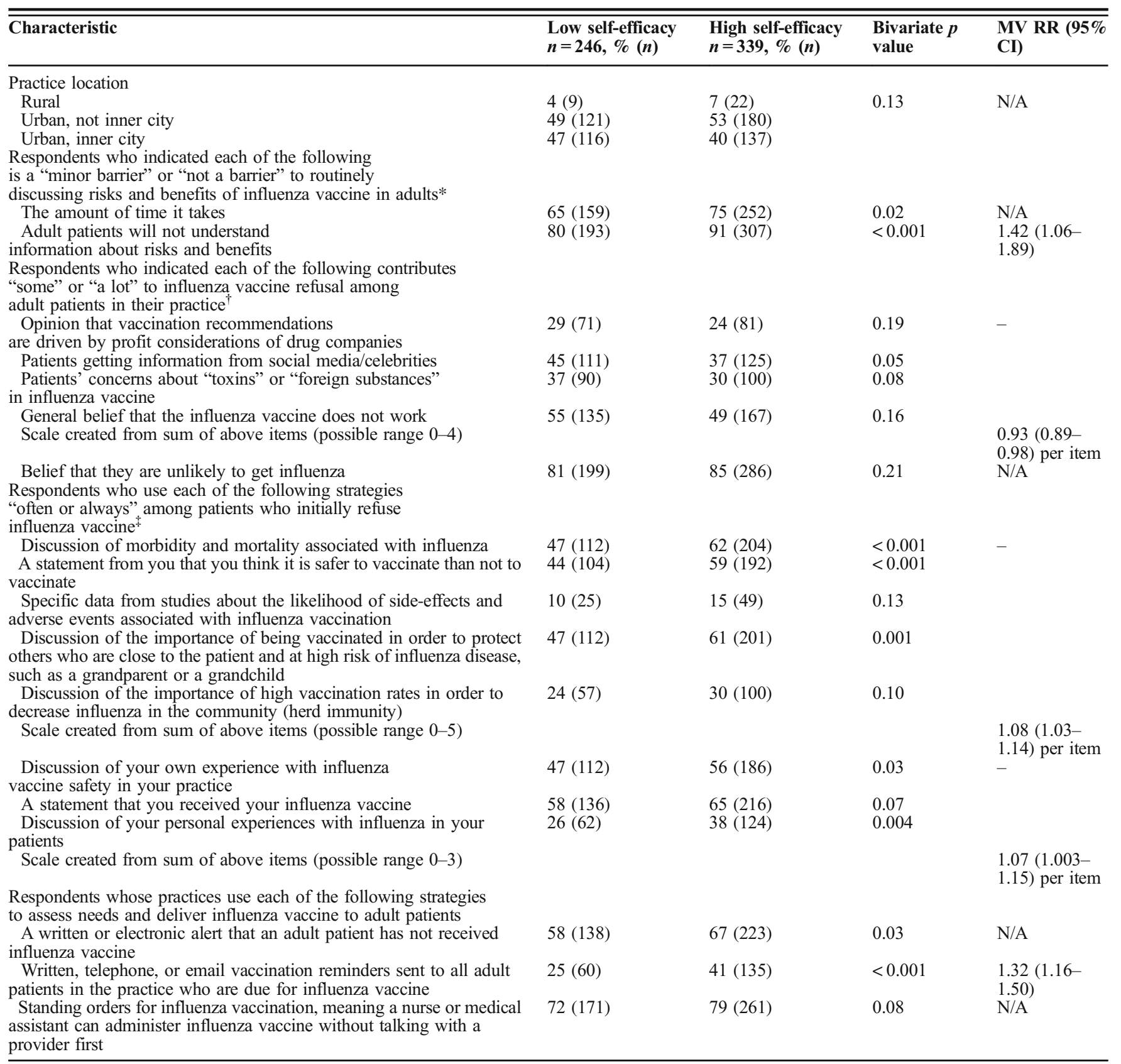

Factor analysis was performed to group similar items in the categories of perceived reasons for patient refusal and provider communication strategies, because each of these categories had $\geq 5$ items significantly associated with the outcome in bivariate analysis. Factor analysis resulted in one scale for reasons for patient refusal including four items reflecting patient suspicions or misinformation about influenza vaccine (Cronbach's alpha $=0.70)$ and two scales for provider communication strategies with one scale of five items reflecting fact-based strategies (Cronbach's alpha $=0.66)$ and a second scale of three items reflecting personal experience-based strategies (Cronbach's alpha $=0.65$ ). Multivariable model also included physician specialty, even though it was non-significant ( $p=0.14$, data not shown)

*Other response categories were "somewhat of a barrier" or a "major barrier," collapsed for dichotomous categorical comparison

"Other response categories were "a little" or "not at all," collapsed for dichotomous categorical comparison

"Other response categories were "never," "rarely," or "sometimes," collapsed for dichotomous comparison

increased their willingness to receive influenza vaccine. ${ }^{22,23}$ Nuanced messaging that focuses on the benefits of protecting people close to adult patients warrants additional investigation in controlled studies as a potential strategy to increase vaccine uptake.

Other strategies that physicians perceived to be most effective included statements about personal experience with influenza vaccine and disease. Multivariable analysis showed that use of these personal experience-based communication strategies was associated with high provider self-efficacy. Although discussion of a physician's personal experience caring for patients with influenza was perceived to be an effective strategy and was used more often by providers with high self-efficacy, it was among the less frequently used 
strategies overall. This emphasis on personal experiencebased messaging from the physician may reflect the fact that receiving a provider recommendation is associated with vaccination. ${ }^{7-9,24,25}$ Additional study is needed to determine whether incorporating the personal provider experience is more effective than a generic recommendation and whether increased use of those strategies perceived to be effective impacts vaccine acceptance among patients.

The top two factors identified as barriers to physicians discussing influenza vaccination with adult patients were other health issues taking precedence and the time needed to discuss the vaccine. These findings are consistent with qualitative and survey studies among Canadian, Australian, and US physicians that have previously identified time and competing priorities as barriers to adult vaccination, including influenza. ${ }^{26-28}$ To address time-related barriers, time-efficient communication strategies for influenza vaccination could include messages or techniques that could be implemented outside of the patient-physician encounter. Some have suggested use of text messages as a way to improve communication of influenza vaccination recommendations. ${ }^{29}$ Others have shown use of a web-based intervention may be an effective way to communicate with patients about pediatric vaccinations which moves the conversation beyond the time constraints of the office visit. ${ }^{30}$ Noting that provider recommendation for influenza vaccination is associated with vaccine uptake, interventions such as social media, text messages, or other patient reminders may be more effective if linked to a recommendation from the patient's physician. Solutions to the challenge of competing patient concerns and other diagnoses are urgently needed because patients with underlying medical problems often face greater risk of morbidity from influenza and stand to benefit most from vaccination.

One approach to optimizing adult influenza vaccination outside of the patient-physician encounter is to use practicelevel strategies that have been proven effective in promoting influenza vaccination including reminder/recall systems, standing orders, and use of IIS. ${ }^{10,11,13}$ Our survey found that physicians with higher self-efficacy for convincing patients to accept influenza vaccination were more likely to work in practices that use patient reminders for adult patients who are due for influenza vaccination. It may be that physicians with high self-efficacy place more emphasis on vaccination in general or champion patient-facing practice-level immunization strategies like patient reminders because they are confident in their ability to convince patients to receive the vaccine. Alternatively, having practice-level structures to promote influenza vaccination may enable physicians to feel more successful in communication with their patients. In either case, understanding this relationship may be helpful in guiding effective interventions at the practice and provider levels to improve influenza vaccination uptake.

This study should be interpreted in the context of several limitations. Although our sample was designed to be representative of ACP and AAFP memberships, our findings may not be fully generalizable. The survey reflects physician-reported practices rather than directly observed practices. Survey items related to barriers to communication about influenza vaccination, patient barriers to vaccine uptake, and provider communication strategies may not have included all barriers and strategies, and qualitative methods may be a more robust method to identify barriers and potential solutions. The limited scope of this survey did not incorporate perspectives from patients or nonphysician health care providers nor did it measure vaccination rates or assess specific influenza vaccine formulations.

This study identifies several potential targets for intervention to improve adult influenza vaccination rates. Practicelevel strategies including IIS support and reminder/recall are under-utilized by primary care physicians and should be further promoted and supported in practice. FP and GIM identify a variety of barriers to communication about influenza vaccination and they find few communication strategies to be very effective. Differences in perceived barriers to vaccination and use of particular communication strategies among providers with high self-efficacy for influenza vaccine communication highlight the need for additional studies of how to optimize physician communication of influenza vaccination recommendations.

\section{Acknowledgments:}

The authors would like to thank Bellinda Schoof, MHA, at the AAFP, Arlene Weissman, $P h D$, and Linda Harris from the ACP, and the leaders of the AAFP and ACP for collaborating in the establishment of the sentinel networks in family medicine and general internal medicine. The authors would also like to thank Erin Burns, Alicia Fry, and Mark Thompson from the Centers for Disease Control and Prevention for their assistance in developing the survey questions. In addition, we would like to thank all general internists and family medicine physicians in the networks for participating and responding to this survey.

Funding Sources: This publication was supported by Cooperative Agreement Number 1 U01 IP000849-03, funded by the Centers for Disease Control and Prevention.

Corresponding Author: Jessica R. Cataldi, MD, MSCS; Adult and Child Consortium for Health Outcomes Research and Delivery Science, University of Colorado Anschutz Medical Campus and Children's Hospital Colorado, 13123 East 16th Ave, Box 055, Aurora, CO 80045, USA (e-mail: jessica.cataldi@ucdenver.edu). Compliance with Ethical Standards:

Conflict of Interest: The authors declare that they do not have a conflict of interest.

Disclaimer: The contents are solely the responsibility of the authors and do not necessarily represent the official views of the Centers for Disease Control and Prevention or the Department of Health and $\mathrm{Hu}^{-}$ man Services.

\section{REFERENCES}

1. Thompson WW, Shay DK, Weintraub E, et al. Mortality associated with influenza and respiratory syncytial virus in the United States. Jama. 2003;289(2):179-186. 
2. Garten R, Blanton L, Elal AIA, et al. Update: influenza activity in the United States during the 2017-18 season and composition of the 201819 influenza vaccine. MMWR Morb Mortal Wkly Rep 2018;67(22):634642.

3. Grohskopf LA, Sokolow LZ, Broder KR, et al. Prevention and control of seasonal influenza with vaccines: recommendations of the Advisory Committee On Immunization Practices - United States, 2017-18 influenza season. MMWR Recomm Rep 2017;66(2):1-20.

4. Office of Disease Prevention and Health Promotion DoHaHS. Healthy People 2020: Immunization and Infectious Diseases. 2018; http://www. healthypeople.gov/2020/topics-objectives/topic/immunization-and-infectious-diseases/objectives. Accessed 6 May 2019.

5. Estimates of influenza vaccination coverage among adults-United States, 2017-18 flu season. Centers for Disease Control and Prevention, National Center for Immunization and Respiratory Diseases (NCIRD). 2018 ; https: / / www.cdc.gov/flu / fluvaxview / coverage1718estimates.htm. Accessed 6 May 2019.

6. Lu PJ, Srivastav A, Santibanez TA, et al. Knowledge of influenza vaccination recommendation and early vaccination uptake during the 2015-16 season among adults aged $>/=18$ years - United States. Vaccine 2017;35(34):4346-4354.

7. Arriola CS, Mercado-Crespo MC, Rivera B, et al. Reasons for low influenza vaccination coverage among adults in Puerto Rico, influenza season 2013-2014. Vaccine 2015;33(32):3829-3835.

8. Nowak GJ, Sheedy K, Bursey K, Smith TM, Basket M. Promoting influenza vaccination: insights from a qualitative meta-analysis of 14 years of influenza-related communications research by U.S. Centers for Disease Control and Prevention (CDC). Vaccine 2015;33(24):2741-2756.

9. Malosh R, Ohmit SE, Petrie JG, Thompson MG, Aiello AE, Monto AS Factors associated with influenza vaccine receipt in community dwelling adults and their children. Vaccine 2014;32(16):1841-1847.

10. Jacobson Vann JC, Jacobson RM, Coyne-Beasley T, Asafu-Adjei JK, Szilagyi PG. Patient reminder and recall interventions to improve immunization rates. Cochrane Database Syst Rev 2018;1:CD003941.

11. Groom H, Hopkins DP, Pabst $\mathbf{L J}$, et al. Immunization information systems to increase vaccination rates: a community guide systematic review. J Public Health Manag Pract 2015;21(3):227-248.

12. U.S. Department of Health and Human Services. National Center for Immunization and Respiratory Diseases. 2017. https://www.cdc.gov/ flu/fluvaxview/coverage-1617estimates.htm. Accessed 6 May 2019.

13. National Vaccine Advisory C. Recommendations from the National Vaccine Advisory committee: standards for adult immunization practice. Public Health Rep 2014;129(2):115-123.

14. Centers for Disease Control and Prevention. Standards for Adult Immunization Practice. 2018; https://www.cdc.gov/vaccines/hcp/ adults/for-practice/standards/index.html. Accessed 6 May 2019.

15. Crane LA, Daley MF, Barrow J, et al. Sentinel physician networks as a technique for rapid immunization policy surveys. Eval Health Prof 2008;31(1):43-64.
16. Brtnikova M, Crane LA, Allison MA, Hurley LP, Beaty BL, Kempe A. A method for achieving high response rates in national surveys of U.S. primary care physicians. PLoS One 2018;13(8):e0202755.

17. Dillman DA, Smyth JD, Christian LM. Internet, Phone, Mail, and MixedMode Surveys: the Tailored Design Method. John Wiley \& Sons; 2014.

18. Atkeson LR, Adams AN, Bryant LA, Zilberman L, Saunders KL Considering mixed mode surveys for questions in political behavior: using the Internet and mail to get quality data at reasonable costs. Polit Behav 2011;33(1):161-178.

19. McMahon SR, Iwamoto M, Massoudi MS, et al. Comparison of e-mail, fax, and postal surveys of pediatricians. Pediatrics 2003;111(4):e299-e303.

20. BanduraA. Self-efficacy. The Corsini Encyclopedia of Psychology. 2010:13.

21. Bandura A. The explanatory and predictive scope of self-efficacy theory. $\mathrm{J}$ Soc Clin Psychol 1986;4(3):359-373.

22. Bednarczyk RA, Chu SL, Sickler H, Shaw J, Nadeau JA, McNutt LA Low uptake of influenza vaccine among university students: evaluating predictors beyond cost and safety concerns. Vaccine 2015;33(14):16591663.

23. Logan J, Nederhoff $\mathbf{D}$, Koch B, et al. What have you HEARD about the HERD? Does education about local influenza vaccination coverage and herd immunity affect willingness to vaccinate? Vaccine 2018;36(28):4118-4125.

24. Nowalk MP, Zimmerman RK, Shen S, Jewell IK, Raymund M. Barriers to pneumococcal and influenza vaccination in older community-dwelling adults (2000-2001). J Am Geriatr Soc 2004;52(1):25-30.

25. MacDougall DM, Halperin BA, Mackinnon-Cameron D, et al. The challenge of vaccinating adults: attitudes and beliefs of the Canadian public and healthcare providers. BMJ Open 2015;5(9):e009062.

26. Omura J, Buxton J, Kaczorowski $\mathbf{J}$, et al. Immunization delivery in British Columbia: perspectives of primary care physicians. Can Fam Physician 2014;60(3):e187-193.

27. Ridda I, Lindley IR, Gao Z, McIntyre P, Macintyre CR. Differences in attitudes, beliefs and knowledge of hospital health care workers and community doctors to vaccination of older people. Vaccine 2008;26(44):5633-5640

28. Szilagyi PG, Shone LP, Barth R, et al. Physician practices and attitudes regarding adult immunizations. Prev Med 2005;40(2):152-161.

29. Phillips AL, Kumar D, Patel S, Arya M. Using text messages to improve patient-doctor communication among racial and ethnic minority adults: an innovative solution to increase influenza vaccinations. Prev Med 2014:69:117-119.

Publisher's Note Springer Nature remains neutral with regard to jurisdictional claims in published maps and institutional affiliations. 